\title{
A Preliminary Study on Continuous Authentication Methods for Photoplethysmographic Biometrics
}

\author{
Angelo Bonissi, Ruggero Donida Labati, Luca Perico, Roberto Sassi, Fabio Scotti, Luca Sparagino \\ Department of Computer Science \\ Università degli Studi di Milano \\ Milano, 20122, Italy. \\ \{ruggero.donida, roberto.sassi, fabio.scotti\}@unimi.it \\ \{angelo.bonissi, luca.perico2, luca.sparagino\}@studenti.unimi.it
}

\begin{abstract}
Recent studies in biometrics focus on one dimensional physiological signals commonly acquired in medical applications, like electrocardiogram (ECG), electroencephalograms (EEG), phonocardiogram (PCG), and photoplethysmogram (PPG). In this context, an important application is in continuous authentication scenarios since physiological signals are frequently captured for long time periods in order to monitor the health status of the patients.

With respect to other physiological signals, PPG data present some advantages since they can be captured using widely diffused, comfortable, and low-cost sensors.

In this paper, we present a feasibility study on continuous authentication techniques based on PPG signals. A biometric recognition method based on a correlation approach is first presented. Then, the accuracy of this method is evaluated on different datasets describing signals of variable time duration. Finally, the performance of continuous enrollment strategies are investigated.

The obtained results suggest that PPG signals present sufficient discriminability to be used in biometric applications that do not require very high accuracy. Moreover, the use of continuous enrollment strategies can improve the performance of continuous authentication systems.
\end{abstract}

\section{INTRODUCTION}

Biometrics is defined by the International Organization for Standardization (ISO) as "the automated recognition of individuals based on their behavioral and biological characteristics" [1]. Some examples of commonly used characteristics, called biometric traits, are fingerprint, iris, palmprint, DNA, voice, and gait.

Recent studies in biometrics focus on the use of onedimensional physiological signals in recognition applications [2]. In particular: the electrocardiogram (ECG) [3], electroencephalogram (EEG) [4], phonocardiogram (PCG) [5], and photoplethysmogram (PPG) [6-10].

These physiological signals are commonly acquired and stored as part of the healthcare delivery process. In applicative contexts like hospitals and body sensor networks [2], the use of biometric systems based on physiological signals should therefore permit to perform recognitions based on existing sensors, without introducing additional costs. Another important characteristic of the one-dimensional physiological signals is that they can be used in continuous authentication systems [11-13] since they can be captured for long time periods without requiring any user's cooperation. Other advantages of these signals are that they can be acquired from all the individuals and can be obtained only from living people [14].

With respect to the other commonly used one-dimensional physiological signals, PPG data offer important advantages. The acquisition of PPG signals, in fact, is performed in a comfortable manner since the samples are captured using pulse oximeters [15], which are non-invasive devices for monitoring the hemoglobin saturation, and are usually attached to the earlobe or fingertip. Moreover, the sensor placement does not require the use of gels and the acquisition devices are widely diffused in medical applications. Other advantages are that pulse oximeters are usually less expensive and smaller in size with respect to the acquisition devices used for the ECG, EEG, and PCG signals. An example of PPG acquisition is shown in Fig. 1.

In the literature, there are some preliminary studies on the use of the PPG as a biometric trait [6-10]. The performed analyses, however, are based on simple features and on small datasets.

In this paper, we present a study on biometric recognition techniques based on PPG signals. In particular, we consider continuous authentication applications.

In this context, we propose a novel recognition technique inspired to the ones used for the analysis of ECG signals. First, a preprocessing step is applied in order to enhance the distinctive characteristics of the PPG signal. The second step is the feature extraction and computes a template describing a defined number of distinctive heartbeats. Finally, the matching is performed by using a correlation-based algorithm.

Experimental results prove that PPG signals present sufficient discriminability to be used in biometric recognition systems. Moreover, the analysis of signals related to long time periods suggests that PPG can effectively be used to perform continuous authentications if continuous enrollment strategies are adopted.

The paper is structured as follows. Section II describes the 
(a)

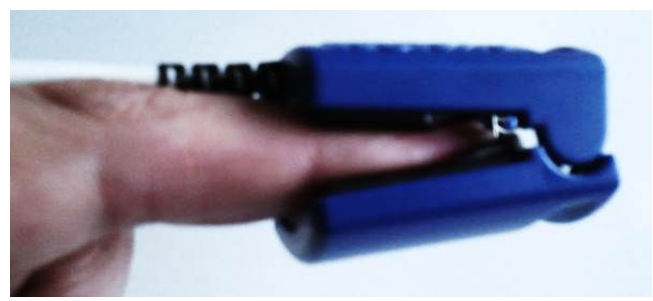

(b)

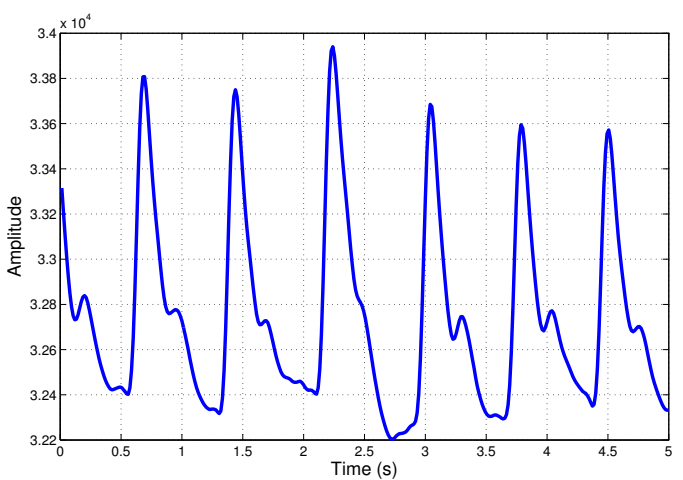

Fig. 1. Acquisition of a photoplethysmogram (PPG) using a pulse oximeter attached to the fingertip: (a) acquisition procedure; (b) obtained signal.

state of the art on the use of PPG signals in biometric systems. Section III presents the implemented recognition methods. The experimental results are detailed in Section IV. Finally, Section $\mathrm{V}$ presents conclusions and final remarks.

\section{RELATED WORKS}

In the literature, there are studies on biometric recognition methods based on different kinds of one-dimensional physiological systems [2]. Most of them evaluate characteristics of ECG [3] and EEG [4] signals. At the best of our knowledge, there are only preliminary studies on PCG [5] and PPG [6-10] signals.

With respect to the other mentioned physiological signal, the use of PPG data has the advantage of requiring less expensive, smaller, and more comfortable acquisition sensors.

In the context of biometric systems, PPG signals have been mainly studied in order to design liveness detection techniques $[16,17]$.

The studies on biometric recognition methods based on PPG signals have been performed by using small sample datasets and algorithms inspired to medical analysis techniques. The method presented in [6] computes the templates by using four features: the peak number, upward slope, downward slope, and time interval. The used matching function is based on the Euclidean distance between the templates. The same features are used in [9], but the matching technique is based on a fuzzy system. The study presented in [7] uses feature sets composed by the values of the local maximum, minimum, and inflection points of PPG pulses in order to evaluate if samples of genuine and impostor individuals present sufficient discriminability for being used in biometric recognition systems. The method described in [8] performs biometric recognitions by applying the linear discriminant analysis (LDA) technique and using nearest neighbor classifiers. Another study is presented in [10] and it proposes the use of PPG-based biometric systems in body area sensor networks and mobile healthcare systems. A study on acquisition sensors specifically designed for biometric recognition is presented in [15].

At the best of our knowledge, there are no studies on the use of PPG signals in continuous authentication systems and the only continuous authentication techniques based on onedimensional physiological signals are described in $[12,13]$. These studies are based on commercial biometric recognition systems. The method presented in [12] performs authentications based on ECG signals every 35 heartbeats and the one detailed in [13] uses time intervals of $5 \mathrm{~s}$.

In the literature, there are also studies on different biometric traits that can be used in continuous authentication systems [11]. In particular, the method presented in [18] evaluates the face characteristics in scenarios in which the users are working in front to a computer. A similar scenario is considered in [19], but the presented biometric technique is also based on less distinctive characteristics obtained analyzing soft biometric traits. The study described in [20] is also based on a multimodal biometric technique, but considers the face and fingerprint traits. Since samples of these traits can be captured at different time intervals, a holistic strategy is proposed to fuse the matching scores. A different data fusion strategy for continuous authentication systems is presented in [21], and is based on fuzzy systems.

\section{THE PROPOSED METHOD}

The proposed method permits to perform biometric recognitions based on PPG signals in a simple and fast manner. We have also implemented and evaluated more complex techniques, obtaining similar results in terms of accuracy, but increasing the needed computational time.

Similarly to most of the biometric systems in the literature, the proposed method can be divided into the sequent steps: acquisition, signal preprocessing, feature extraction, matching.

\section{A. Acquisition}

The acquisition process is performed with a NONIN Xpod pulse oximeter [22], commonly used for medical diagnoses.

In the context of continuous authentication systems, the PPG is continuously acquired and a sample is stored every $t \mathrm{~s}$. The considered samples have a fixed time duration of $t \mathrm{~s}$.

Pulse oximeters, however, are sensitive to user's movements since the acquisition device needs a continuous contact with the user's skin. For this reason, it is necessary to define a strategy for discarding poor quality samples. In this work, we adopt a basic automatic procedure to discard samples with insufficient quality. The data generated by the used pulse oximeter include a binary signal representing the presence of possible acquisition errors at every time instance. If a sample presents at least an error state, it is considered as a failure 
to acquire (FTA). An example of a $20 \mathrm{~s}$ sample acquired during the user's movement and the corresponding error states generated by the acquisition device are shown in Fig. 2. It is possible to observe that the portions of the signal near to the errors detected by the pulse oximeter present spikes and irregular shapes caused by the noise introduced by the displacement of the sensor from the finger surface.

\section{B. Signal preprocessing}

The proposed method does not use complex preprocessing algorithms.

The only executed task consists in the signal's baseline normalization, which is performed by applying a third order high-pass Butterworth filter with cutoff frequency $f_{c}$ [23].

\section{Feature extraction}

This step computes a template $T$ composed by a variable number of distinctive heartbeats of the sample signal $S$. The maximum number of heartbeat signals composing a template $T$ is equal to $n$.

The first task consists in the signal segmentation, which permits to obtain a matrix $H$ in which the points pertaining to every heartbeat signal are stored in a distinct row. The peaks are detected using a modified Pan-Tompkins algorithm. For a given peak instant $j$, the heartbeat signal $i$ is computed as:

$$
H\left(i, 1 \ldots t_{1}+t_{2}\right)=S\left(j-t_{1} \ldots i+t_{2}\right),
$$

where $t_{1}$ and $t_{2}$ are constant values empirically estimated on the considered dataset.

The biometric template is finally obtained by applying an iterative algorithm. For each iteration, the signal $\bar{S}$ representing the mean heartbeat is computed as:

$$
\bar{S}(j)=\sum_{i=1}^{m}(H(i, j)) / m,
$$

where $m$ is the number of heartbeats present in the matrix $H$.

A vector $C$ representing the maximum cross-correlation values between every heartbeat of $H$ and the mean heartbeat $\bar{S}$ is computed. A fixed number $n$ of heartbeats corresponding to the $n$ maximum values of $C$ are stored in the template matrix $T$. If the correlation value of a heartbeat is less than an empirically estimated threshold $t_{c}$, the related signal is removed from the template $T$.

In order to reduce the noise, this algorithm is repeated two times. The second iteration aims to delete possible noisy heartbeats and considers $H=T$. The number $n$ is equal for every iteration.

\section{Matching}

This step computes a similarity score $s$ between two templates $T_{A}$ and $T_{B}$ composed by $n_{A}$ and $n_{B}$ signals of hearbeats, respectively.

A matrix $M$, composed by $n_{A} n_{B}$ elements, is computed by calculating the maximum cross-correlation between every signal pertaining to $T_{A}$ and every signal pertaining to $T_{B}$.

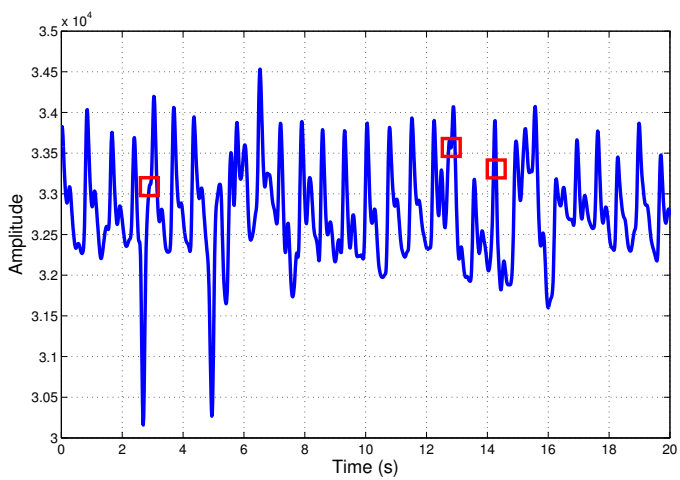

Fig. 2. Example of $20 \mathrm{~s}$ sample captured during user's movements and considered as a failure to acquire (FTA). The red squares represent error states of the pulse oximeter. It is possible to observe the presence of spikes and noisy regions.

An example of matrix $M$ obtained from a genuine identity comparison of templates composed by two heartbeats is shown in Fig. 3.

Finally, the matching score $s$ can be obtained by applying different data fusion strategies to the similarity values of $M$. In this study, we have considered these simple techniques.

- Algorithm A: $s=\operatorname{mean}(M)$.

- Algorithm B: $s=\operatorname{median}(M)$.

- Algorithm C: $s=75$ th percentile $(M)$.

- Algorithm D: $s=90$ th percentile $(M)$.

- Algorithm E: $s=95$ th percentile $(M)$.

- Algorithm F: $s=\max (M)$.

\section{EXPERIMENTAL RESULTS}

In order to analyze the performance of the proposed method, we have first evaluated the recognition accuracy in different operative conditions and then analyzed the feasibility of a continuous authentication systems based on PPG signals.

The used datasets have been captured in our laboratory. At the best of our knowledge, in fact, there are no public datasets of PPG signals with sufficient number of samples to properly evaluate a biometric systems.

The parameters used for testing the recognition method are: $f_{c}=0.1 \mathrm{~Hz}, n=16, t_{1}=0.12 \mathrm{~s}, t_{2}=0.24$, and minimum correlation threshold for the template computation is $t_{c}=0.9$.

\section{A. Tuning of the recognition algorithms}

In order to search the best configuration of the implemented recognition algorithms, we have first evaluated the accuracy of the biometric system on a dataset of short time signals captured in our laboratory.

We have collected a set of 44 PPG signals from 44 individuals by using a NONIN Xpod pulse oximeter [22] with a finger sensor. Every acquired signal has a duration of 2 minutes, a resolution of 16 bit, and a sampling frequency of $75 \mathrm{~Hz}$.

In order to evaluate the capability of the proposed recognition method of working with sample signals of different duration, three datasets have been created from the acquired 
(a)
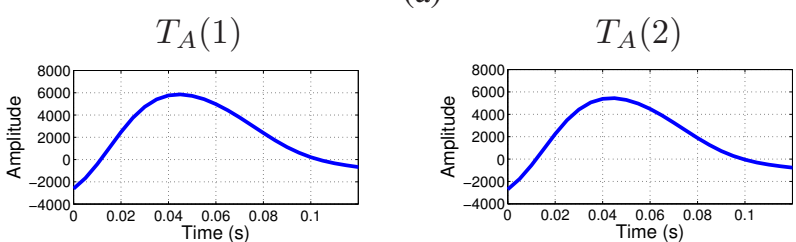

(b)
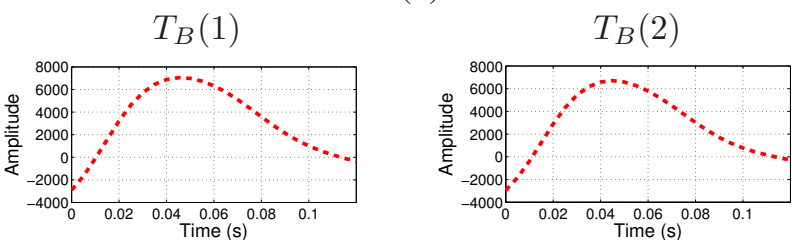

(c)

\begin{tabular}{l|l|l|} 
& \multicolumn{1}{c}{$T_{B}(1)$} & $T_{B}(2)$ \\
\cline { 2 - 3 }$T_{A}(1)$ & 0.994 & 0.997 \\
\cline { 2 - 3 }$T_{A}(2)$ & 0.985 & 0.990 \\
\cline { 2 - 3 } & &
\end{tabular}

Fig. 3. Example of similarity matrix $M$ obtained from a genuine identity comparison between the templates $T_{A}$ and $T_{B}$, which are composed by two heartbeat signals $(n=2)$ : (a) template $T_{A}$; (a) template $T_{B}$; similarity matrix $M$.

TABLE I

DATASETS CREATED FROM 2 MINUTES SIGNALS.

\begin{tabular}{ccccc}
\hline \hline Dataset & $\begin{array}{c}\text { Sample } \\
\text { duration } t\end{array}$ & $\begin{array}{c}\text { Number of } \\
\text { samples }\end{array}$ & $\begin{array}{c}\text { Genuine } \\
\text { comparisons }\end{array}$ & $\begin{array}{c}\text { Impostor } \\
\text { comparisons }\end{array}$ \\
\hline $\mathrm{DB}_{20}$ & $20 \mathrm{~s}$ & 242 & 1154 & 57168 \\
$\mathrm{DB}_{20}$ & $30 \mathrm{~s}$ & 158 & 454 & 24352 \\
$\mathrm{DB}_{40}$ & $40 \mathrm{~s}$ & 116 & 224 & 13116 \\
\hline \hline
\end{tabular}

set of signals. The characteristics of these datasets are depicted in Table I.

We have evaluated the accuracy of the recognition method on the test datasets by using the implemented data fusion strategies. In all the performed evaluations, Algorithm F obtained the best accuracy. The DET curves obtained by Algorithm F on the considered datasets are shown in Fig. 4, while Table II reports the false match rate (FMR) and false non-match rate (FNMR) in different working points.

From Fig. 4 and Table II, it is possible to observe that the discriminability of PPG signals in short time periods can be considered as satisfactory for many biometric applications. Moreover, the obtained results show that the best accuracy has been obtained on $\mathrm{DB}_{40}$, with an equal error rate (EER) of $5.29 \%$. The accuracy increasing with respect to the other datasets should be due to the better capability of the proposed method of reducing the effects of the noise due to user's movements. However, the smaller number of performed comparisons can also influence the obtained performances.

\section{B. Preliminary test in continuous authentication scenarios}

We have then evaluated the accuracy of the biometric recognition method on a dataset composed by longer time signals.

Similarly to the previous test, we have collected a set of

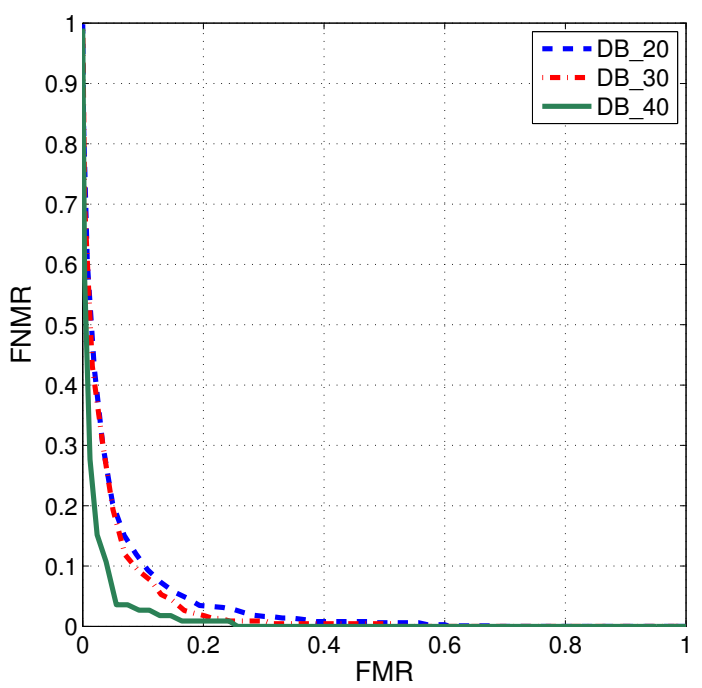

Fig. 4. DET curves obtained by realized method by using Algorithm F on $\mathrm{DB}_{20}, \mathrm{DB}_{20}$, and $\mathrm{DB}_{40}$.

TABLE II

RESULTS OF THE REALIZED METHOD BY USING ALGORITHM F ON DIFFERENT DATASETS OF SHORT TIME SIGNALS.

\begin{tabular}{llccc}
\hline \hline DET point & $\mathrm{DB}_{20}$ & $\mathrm{DB}_{20}$ & $\mathrm{DB}_{40}$ \\
\hline EER (\%) & 10.06 & 8.34 & 5.29 \\
FMR (\%) & FNMR $=5 \%$ & 15.08 & 11.89 & 10.71 \\
FMR (\%) & FNMR $=10 \%$ & 9.36 & 5.29 & 2.68 \\
FMR (\%) & FNMR $=15 \%$ & 5.72 & 2.20 & 1.79 \\
FNMR (\%) & FMR $=5 \%$ & 15.10 & 11.03 & 5.00 \\
FNMR (\%) & FMR $=10 \%$ & 8.84 & 5.57 & 5.00 \\
FNMR (\%) & FMR $=15 \%$ & 6.86 & 3.86 & 3.34 \\
\hline \hline
\end{tabular}

14 PPG signals from 14 individuals by using a NONIN Xpod pulse oximeter. The duration of each acquisition is 15 minutes.

Considering the previously obtained results, the collected signals have then been divided into samples of $40 \mathrm{~s}$, obtaining the dataset $\mathrm{DB}_{15 \mathrm{~m}}$.

The results obtained by the best configuration of the proposed method (Algorithm $\mathrm{F}$ ) on $\mathrm{DB}_{15 \mathrm{~m}}$ are reported in Fig. 5 and Table III. It is possible to observe that the obtained accuracy is inferior with respect the one achieved on $\mathrm{DB}_{40}$. The obtained EER, for example, is equal to $13.47 \%$.

The performance decreasing can be related to the time variability of the biometric trait with the used features.

In order to evaluate this phenomena, we have analyzed the performance of the presented algorithms in an ideal continuous authentication scenario in which verifications are performed at fixed time intervals and the enrolled templates are updated after every verification. Differently from traditional procedures used for the accuracy evaluation of biometric recognition algorithms, which do not consider the time passed between biometric acquisitions [24], this test permits to analyze the stability and persistency of the used features during a defined time interval. In this test, we have not used samples considered as FTA since it only aims to evaluate the persistency of PPG 


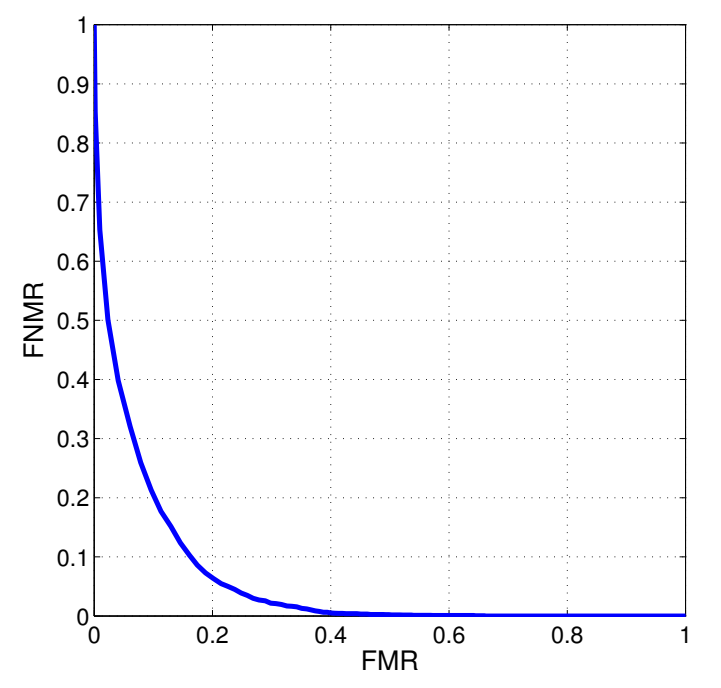

Fig. 5. DET curve obtained on $\mathrm{DB}_{15 \mathrm{~m}}$. It is possible to observe a performance decreasing with respect to the results obtained on $\mathrm{DB}_{40}$, which is due to a low durability of the biometric features.

TABLE III

RESUlts of THE REALIZED METHOD B Y USING ALGORITHM F ON DB $15 \mathrm{~m}$.

\begin{tabular}{lc}
\hline \hline \multicolumn{1}{l}{ DET point } & $\mathrm{DB}_{15 \mathrm{~m}}$ \\
\hline EER (\%) & 13.47 \\
FMR (\%) FNMR $=5 \%$ & 32.06 \\
FMR (\%) FNMR $=10 \%$ & 17.71 \\
FMR (\%) FNMR $=15 \%$ & 10.30 \\
FNMR (\%) $\quad$ FMR $=5 \%$ & 22.56 \\
FNMR (\%) $\quad$ FMR $=10 \%$ & 16.07 \\
FNMR (\%) $\quad$ FMR $=15 \%$ & 12.97 \\
\hline \hline
\end{tabular}

signals.

All the possible impostor identity comparisons have been computed. For each time instant $j$, we have computed the genuine matching score between the template $T_{(i, j)}$ and the template $T_{\left(i, j-\Delta_{t}\right)}$ obtained at the time instant $j-\Delta_{t}$ :

$$
G_{(i, j)}=\operatorname{matching}\left(T_{(i, j)}, T_{\left(i, j-\Delta_{t}\right)}\right) .
$$

The EER obtained with different values of $\Delta_{t}$ are reported in Fig. 6 and Table IV. The error obtained in other working points is not reported since the accuracy evaluations consider only small numbers of genuine identity comparisons.

From Fig. 6 and Table IV, it can be observed that the accuracy of the recognition method is strictly related to the time interval between the considered genuine comparisons $\Delta_{t}$. The obtained EER values, in fact, decrease from $9 \%$ to $14.5 \%$ in $320 \mathrm{~s}$. The accuracy decreasing, anyway, is not linear since poor quality samples and physiological changes could influence the obtained results.

The performed preliminary tests suggest that the used features have not sufficient durability for being used in traditional biometric recognition scenarios. PPG signals, anyway, should be used to perform continuous authentications by adopting continuous enrollment strategies.

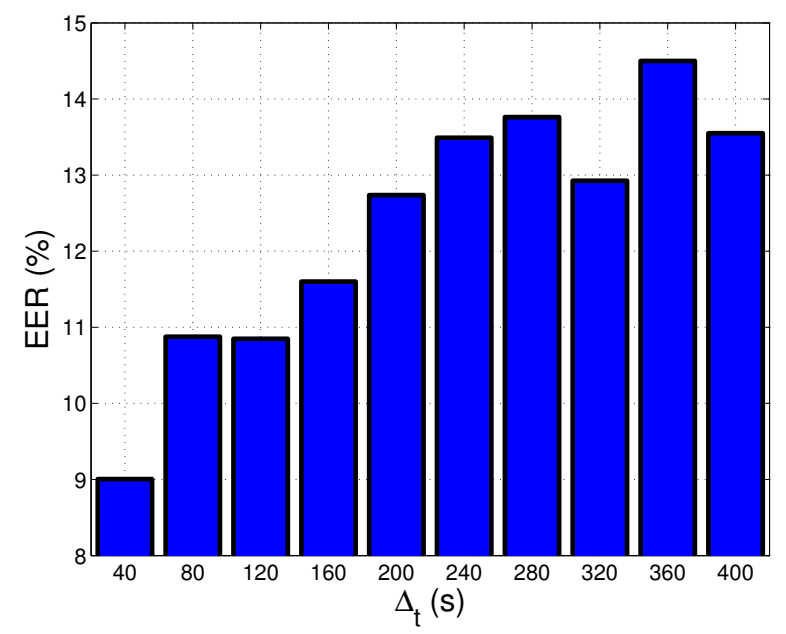

Fig. 6. EER value in a continuous authentication system with continuous enrollment every $\Delta_{t} \mathrm{~s}$. The obtained accuracy decreases proportionally to the values of $\Delta_{t}$.

TABLE IV

EER VALUE IN A CONTINUOUS AUTHENTICATION SYSTEM WITH CONTINUOUS ENROLLMENT EVERY $\Delta_{t} \mathrm{~S}$.

\begin{tabular}{cccc}
\hline \hline $\begin{array}{c}\Delta_{t} \\
(\mathbf{s})\end{array}$ & $\begin{array}{c}\text { Genuine } \\
\text { comparisons }\end{array}$ & $\begin{array}{c}\text { Impostor } \\
\text { comparisons }\end{array}$ & $\begin{array}{c}\text { EER } \\
(\boldsymbol{\%})\end{array}$ \\
\hline 40 & 253 & 73168 & 9.00 \\
80 & 239 & 73168 & 10.88 \\
120 & 227 & 73168 & 10.85 \\
160 & 220 & 73168 & 11.60 \\
200 & 204 & 73168 & 12.74 \\
240 & 195 & 73168 & 13.49 \\
280 & 179 & 73168 & 13.76 \\
320 & 167 & 73168 & 12.93 \\
360 & 155 & 73168 & 14.50 \\
400 & 145 & 73168 & 13.55 \\
\hline \hline
\end{tabular}

This kind of systems, however, require particular attention in the design of privacy protection strategies since they can be more easily attached by spoofing techniques with respect to traditional biometric recognition methods [25].

\section{CONCLUSION}

This paper have presented a preliminary study on the use of photoplethysmogram (PPG) signals in biometric systems working in continuous authentication scenarios.

The proposed biometric recognition method is based on a simple and fast correlation technique.

The recognition accuracy has been tested on sets of samples with different time duration, with particular attention to the analysis of the relation between the system accuracy and the time passed between genuine acquisitions.

The obtained results suggest that PPG signals have sufficient discriminability to be used in biometric recognition systems. However, the analyzed features present low durability and should therefore be used in continuous authentication systems based on continuous enrollment strategies.

Further studies should regard the implementation of complete continuous authentication systems based on PPG signals. 


\section{ACKNOWLEDGEMENTS}

This work was supported in part by the Italian Ministry of Research within PRIN 2010-2011 project "GenData 2020" (2010RTFWBH).

\section{REFERENCES}

[1] International Organization for Standards, "ISO/IEC JTC1 SC37 Standing Document 2, version 8, Harmonized Biometric Vocabulary ," August 1997.

[2] F. Miao, S.-D. Bao, and Y. Li, Physiological Signal Based Biometrics for Securing Body Sensor Network. InTech, 2012.

[3] I. Odinaka, P.-H. Lai, A. Kaplan, J. O'Sullivan, E. Sirevaag, and J. Rohrbaugh, "Ecg biometric recognition: A comparative analysis," IEEE Transactions on Information Forensics and Security, vol. 7, no. 6, pp. 1812-1824, 2012.

[4] W. Khalifa, A. Salem, M. Roushdy, and K. Revett, "A survey of eeg based user authentication schemes," in Proc. of the 8th Int. Conf. on Informatics and Systems (INFOS), 2012, pp. $55-60$.

[5] F. Beritelli and S. Serrano, "Biometric identification based on frequency analysis of cardiac sounds," IEEE Transactions on Information Forensics and Security, vol. 2, no. 3, pp. 596 - 604, 2007.

[6] Y. Y. Gu, Y. Zhang, and Y. Zhang, "A novel biometric approach in human verification by photoplethysmographic signals," in Proc. of the 4th IEEE Annual Int. Conf. of the Engineering in Medicine and Biology Society (EMBS), 2003, pp. $13-14$

[7] J. Yao, X. Sun, and Y. Wan, "A pilot study on using derivatives of photoplethysmographic signals as a biometric identifier," in Proc. of the 29th IEEE Annual Int. Conf.of the Engineering in Medicine and Biology Society (EMBS), 2007, pp. $4576-4579$.

[8] P. Spachos, J. Gao, and D. Hatzinakos, "Feasibility study of photoplethysmographic signals for biometric identification," in Proc. of the 17th Int. Conf. on Digital Signal Processing (DSP), 2011, pp. 1 - 5.

[9] Y. Y. Gu and Y. Zhang, "Photoplethysmographic authentication through fuzzy logic," in Proc. of the IEEE EMBS Asian-Pacific Conf. on Biomedical Engineering, 2003, pp. 136 - 137.

[10] S.-D. Bao, Y.-T. Zhang, and L.-F. Shen, "Physiological signal based entity authentication for body area sensor networks and mobile healthcare systems," in Proc. of the 27th IEEE-EMBS Annual Int.Conf. of the Engineering in Medicine and Biology Society, 2005, pp. 2455 - 2458.

[11] I. Traore and A. Ahmed, Continuous Authentication Using Biometrics: Data, Models, and Metrics. IGI Global, 2011.

[12] M. Guennoun, N. Abbad, J. Talom, M. Rahman, and K. El-Khatib, "Continuous authentication by electrocardiogram data," in Proc. of the IEEE Toronto Int. Conf. on Science and Technology for Humanity (TICSTH), 2009, pp. 40-42.

[13] R. Matta, J. Lau, F. Agrafioti, and D. Hatzinakos, "Real-time continuous identification system using ecg signals," in Proc. of the 24th Canadian Conference on Electrical and Computer Engineering (CCECE), 2011, pp. $1313-1316$.

[14] B. Biggio, Z. Akthar, G. Fumera, G. L. Marcialis, and F. Roli, "Security evaluation of biometric authentication systems under real spoofing attacks," IET Biometrics, 2012.

[15] Y. Wan, X. Sun, and J. Yao, "Design of a photoplethysmographic sensor for biometric identification," in Proc. of the Int. Conf. on Control, Automation and Systems (ICCAS), 2007, pp. 1897-1900.

[16] P. Reddy, A. Kumar, S. Rahman, and T. Mundra, "A new antispoofing approach for biometric devices," IEEE Transactions on Biomedical Circuits and Systems, vol. 2, no. 4, pp. 328 -337, 2008.

[17] P. Reddy, A. Kumar, S. Rahman, and T. Mundra, "A new method for fingerprint antispoofing using pulse oxiometry," in Proc. of the First IEEE Int. Conf.on Biometrics: Theory, Applications, and Systems (BTAS), 2007, pp. $1-6$.

[18] K. Niinuma and A. K. Jain, "Continuous user authentication using temporal information,” vol. 7667, 2010.

[19] K. Niinuma, U. Park, and A. Jain, "Soft biometric traits for continuous user authentication," IEEE Transactions on Information Forensics and Security, vol. 5, no. 4, pp. 771 - 780, 2010.

[20] T. Sim, S. Zhang, R. Janakiraman, and S. Kumar, "Continuous verification using multimodal biometrics," IEEE Transactions on Pattern Analysis and Machine Intelligence, vol. 29, no. 4, pp. 687 - 700, 2007.
[21] A. Azzini, S. Marrara, R. Sassi, and F. Scotti, "A fuzzy approach to multimodal biometric continuous authentication," Fuzzy Optimization and Decision Making, vol. 7, no. 3, pp. 243 - 256, September 2008.

[22] NONIN Xpod External Pulse Oximetry, http://www.nonin.com.

[23] J. Proakis and D. Manolakis, Digital signal processing. Pearson Prentice Hall, 2007.

[24] M. Gamassi, M. Lazzaroni, M. Misino, V. Piuri, D. Sana, and F. Scotti, "Quality assessment of biometric systems: a comprehensive perspective based on accuracy and performance measurement," IEEE Transactions on Instrumentation and Measurement, vol. 54, no. 4, pp. 1489 - 1496, August 2005.

[25] B. Biggio, Z. Akhtar, G. Fumera, G. Marcialis, and F. Roli, "Security evaluation of biometric authentication systems under real spoofing attacks," IET Biometrics, vol. 1, no. 1, pp. 11 - 24, 2012. 